\title{
Mathematical Modelling of Profiled Haemodialysis: A Simplified Approach
}

\author{
STEPHEN BAIGENT ${ }^{\mathrm{a},}{ }^{*}$, ROBERT UNWIN $^{\mathrm{b}}$ and CHEE CHIT YENG ${ }^{\mathrm{c}}$
}

\begin{abstract}
${ }^{a}$ Centre for Nonlinear Dynamics and Its Applications, University College London, Gower Street, London WC1E 6BT, ${ }^{b}$ Centre for Nephrology and Institute of Urology and Nephrology, Royal Free and University College Medical School Middlesex Hospital, Mortimer Street, London W1N 8AA and 'Formerly Department of Mathematics, Imperial College of Science, Technology and Medicine, Imperial College, 180 Queen's Gate, London, SW7 2BZ
\end{abstract}

\begin{abstract}
For many renal patients with severe loss of kidney function dialysis treatment is the only means of preventing excessive fluid gain and the accumulation of toxic chemicals in the blood. Typically, haemodialysis patients will dialyse three times a week, with each session lasting 4-6 hours. During each session, 2-3 litres of fluid is removed along with catabolic end-products, and osmotically active solutes. In a significant number of patients, the rapid removal of water and osmotically active sodium chloride can lead to hypotension or overhydration and swelling of brain cells. Profiled haemodialysis, in which the rate of water removal and/or the dialysis machine sodium concentration are varied according to a predetermined profile, can help to prevent wide fluctuations in plasma osmolarity, which cause these complications. The profiles are determined on a trial and error basis, and differ from patient to patient. Here we describe a mathematical model for a typical profiled haemodialysis session in which the variables of interest are sodium mass and body fluid volumes. The model is of minimal complexity and so could provide simple guidelines for choosing suitable profiles for individual patients. The model is tested for a series of dialysate sodium profiles to demonstrate the potential benefits of sodium profiling. Next, using the simplicity of the model, we show how to calculate the dialysate sodium profile to model a dialysis session that achieves specified targets of sodium mass removal and weight loss, while keeping the risk of intradialytic complications to a minimum. Finally, we investigate which of the model profiled dialysis sessions that meet a range of sodium and fluid removal targets also predict extracellular sodium concentrations and extracellular volumes that lie within "safe" limits. Our model suggests that improvements in volume control via sodium profiling need to be set against potential problems in maintaining blood concentrations and body fluid compartment volumes within "safe" limits.
\end{abstract}

Keywords: Haemodialysis, Sodium Profiling, Disequilibrium Syndrome, Hypotension, Osmotic Balance

* Corresponding Author. 


\section{INTRODUCTION}

The main function of the kidney is to regulate fluid and electrolyte balance in order to maintain intracellular (IC) and extracellular (EC) fluid volumes and ion compositions within narrow limits. When the kidneys fail to function normally, fluid is retained and several ions and solutes accumulate (mainly in the EC fluid compartment) and may become life threatening, such as potassium and hydrogen ions. While changes in diet and the administration of diuretic drugs can help to offset some of the important fluid and electrolyte problems due to reduced kidney function, in severe cases, dialysis treatment is necessary.

The two main forms of dialysis are haemodialysis and peritoneal dialysis. The latter process, which we do not discuss here, uses the patient's peritoneal membrane, which lines the abdominal cavity and viscera, as the exchange surface to 'filter' the blood by passive osmotic forces with a bathing fluid. Haemodialysis, on the other hand, uses an external and artificial membrane as a filter and surface for exchange. Peritoneal dialysis is a more continuous process, whereas haemodialysis is typically done in 4-6 hourly sessions three times a week. The short duration and intermittent nature of haemodialysis can be problematic. The patient experiences large and acute swings in body fluid volume, and ion and solute composition not normally encountered in health. These changes can result in significant intra- and interdialytic morbidity. This paper first reviews the mechanisms that cause the changes in fluid volumes, and then presents a simple model that may assist renal physicians or technicians to design profiled dialysis therapy that should minimise significant volume changes.

\section{THE DIALYSIS PROCESS}

Haemodialysis is essentially an equilibration ([3],[4]). The patient's blood is pumped (using systemic arterial blood pressure alone or with the assistance of a mechanical pump) around a continuous circuit from an artery to a dialysis machine and then back to a vein. On reaching the dialysis machine, the blood is fed into a hollow-fibre dialyser, a small cylinder containing many thousands of minute hollow fibres with small pores. On the other side of these pores, in contraflow, is pumped an acetate- or bicarbonate-based fluid of prescribed composition known as the dialysate. In standard dialysis treatment the composition of the dialysate is maintained constant. As the blood traverses the dialyser, water and solutes (ions and nitrogenous products of normal metabolism) diffuse both ways across the porous membrane in accordance with concentration gradients and an applied hydraulic pressure. On reaching the end of the dialyser, the blood is pumped back into the body. If continued indefinitely, and without pressure gradients, the blood concentrations would eventually equilibrate (osmotically) with those of the constant dialysate. In practice dialysis sessions are time constrained, although equilibrium may be achieved for some solutes. This leads to an important point: solutes and solvent (water) are removed at different rates, and furthermore their removal may be coupled, which is the main reason for intradialytic morbidity. We expand on this in more detail below, but before doing so, we list the key substances that dialysis therapy aims to remove.

(1) Water: Most patients are anuric or produce very little urine, so that dialysis is their only means of removing excess fluid. Each patient has a theoretical $d r y$ weight an index of total body water volume, which is estimated as 0.58 of their body weight in kilograms [5]. Thus, a patient weighing $65 \mathrm{~kg}$ will have a total body water fluid volume of 37.7 litres. In a patient who complies with dietary guidelines, fluid gain will typically be 2-3 litres (as kilogram weight). This will be the target for fluid removal during a single dialysis session.

(2) Sodium: The balance of sodium, present mainly as sodium chloride, is an important aim of dialysis. Sodium, which is relatively impermeable to cell membranes, is the most abundant EC osmolyte. Changes in sodium concentration, and therefore osmolarity, shifts body water to regions of higher osmolarity. Large shifts of water, as we will describe later, can lead to major intra- and 
interdialytic changes in fluid distribution between the IC and EC compartments.

(3) Urea: Urea is a normal product of protein catabolism and is excreted by the kidneys. At high concentrations it may affect the stability of proteins, but in kidney failure its accumulation in the blood is also a marker of the retention of other more toxic products of nitrogen metabolism, the so-called 'middle molecules'. Owing to the relatively high permeability of the cell membrane to urea, fluid shifts due to changes in urea concentration are small and can be neglected [5]. However, in some patients there is a pronounced increase in EC urea during the first few hours following dialysis [7], a phenomenon known as urea rebound, and which is due to inter-compartmental equilibration of urea. This can cause a significant drop in IC fluid volume, leading to thirst and the risk of fluid overload from excessive drinking.

(4) Glucose: The control of glucose removal is particularly important in diabetic patients; it may change rapidly during dialysis if its concentration in the dialysate is not properly adjusted.

(5) Potassium: Excitable cells (nerve and muscle) depend for their normal function on a critical balance between the IC and $\mathrm{EC}$ concentrations of potassium. High EC potassium levels can prove fatal by causing a cardiac arrest. Haemodialysis with low dialysate potassium can also be problematic in patients with underlying cardiovascular disease [8], because the reduction in the IC:EC potassium gradient during dialysis can result in increased membrane depolarisation and re-entry cardiac arrhythmias.

For convenience we may think of the total body fluid as being divided between the interstitial (outside cells) and intravascular compartments (which together make up the EC), and the intracellular compartment (IC). In line with other bicompartmental models we can simplify the distribution of body fluids into the extracellular volume (ECV), which is the sum of the interstitial and vascular compartment volumes, and the intracellular volume (ICV). The transport processes involved in dialysis occur between the ICV and ECV, and ECV and the dialysate fluid. These processes include those involving the cell membrane and those involving the hollow-fibre dialyser membrane. For a more comprehensive introduction to the cell physiology and biophysics covered in the following descriptions see [9].

\section{DIALYSER TRANSPORT}

We distinguish between two distinct processes, namely passive diffusion and ultrafiltration.

(1) Passive diffusion is the net movement of solutes and solvent down their respective concentration gradients. By Fick's Law the diffusive flux of a substance is directly proportional to its concentration gradient. The constant of proportionality, the diffusive constant, depends upon the permeability of the medium and is different for each diffusing substance.

(2) Ultrafiltration is the convective transport of solutes in solution across the dialyser membrane. The convective flux of a given solute is proportional to the product of the concentration at source and the flow rate of the solution. Note that the removal of a solute from a compartment by ultrafiltration does not change the solute concentration in the original compartment. In practice the ultrafiltration rate is controlled by adjusting the applied transmembrane pressure gradient.

\section{CELLULAR TRANSPORT}

There are three processes to describe, as shown in Figure 1.

(1) Passive diffusion (as above).

(2) Osmosis. This is passive diffusion of water down its concentration gradient. The osmolarity (in osmoles) of a solute in a given volume is determined by the total number of solute particles displacing the water, and is calculated by summing up the molar concentrations of each solute. When a molecule ionises in solution, such as $\mathrm{NaCl}$, the 


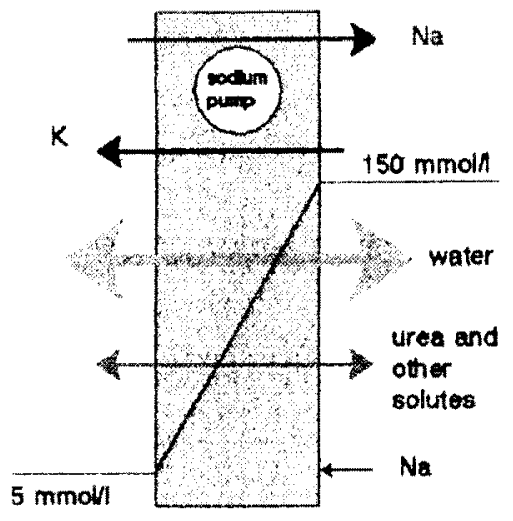

Solute and edvent reneport acrose the patient cell membranes

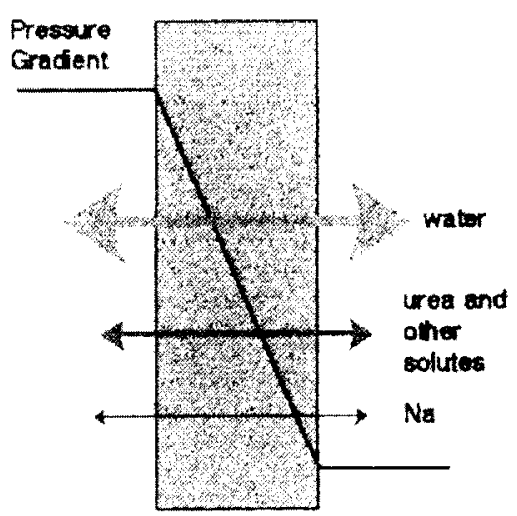

Solute and solvent transpert scross the dialver membrene

FIGURE 1 Transport processes and trans-membrane gradients in and across the cell membrane and dialyser membrane. For the cell membrane, the permeability to sodium is low, whereas for water, urea and other solutes it is high. Although the membrane is slightly permeable to sodium, a constant low internal sodium concentration is maintained by a continuous trans-membrane exchange of sodium and potassium. The dialyser, on the other hand, is freely permeable to water and solutes up to the size of small proteins $(<50,000 \mathrm{Da})$, and filtration is assisted by an imposed hydraulic pressure gradient

molarities of each individual ion must be taken into account. Thus, a 1 molar solution of $\mathrm{NaCl}$ has an osmolarity of 2 osmoles.

(3) Active transport. The factors leading to the establishment of a cell resting potential are the low permeability of the cell membrane to sodium and the presence of membrane-bound sodium pumps that pump out sodium in exchange for potassium. This renders the membrane effectively impermeable to sodium and maintains a low IC sodium concentration. This regulatory mechanism is remarkably robust; even when there are significant changes in the EC ionic composition, the sodium pumps will maintain constant IC sodium mass. In line with [1] and [2], that IC sodium mass is constant, is a key assumption in our model.

\section{INTRADIALYTIC MORBIDITY}

The most common intradialytic complications are symptomatic hypotension and disequilibrium syn- drome. Both are a result of a fall in plasma osmolarity caused by intercompartmental shifts of body fluids.

(1) Symptomatic hypotension is often caused by the rapid removal of fluid without adequate refilling of the EC fluid compartment [5], [9]. The resulting hypovolaemia can be exacerbated by impaired cardiovascular mechanisms that normally compensate for the acute fall in EC and circulating volume. The root cause is usually an excessive ultrafiltration rate leading to hypovolaemia: the rapid fluid loss can be exacerbated by osmotic shifts of water due to the convective transport of osmotically active sodium across the dialysis membrane, as summarised in Figure 2. Although other solutes such as urea and glucose contribute to the osmolarity of the plasma (and the ECV), they enter cells more easily and sodium (as sodium chloride) accounts for around 90\% of plasma osmolarity [5]. Thus sodium balance is critical. (See also [2] for comments on the relative importance of sodium versus urea transport in causing osmotic water shifts). In order to maintain 


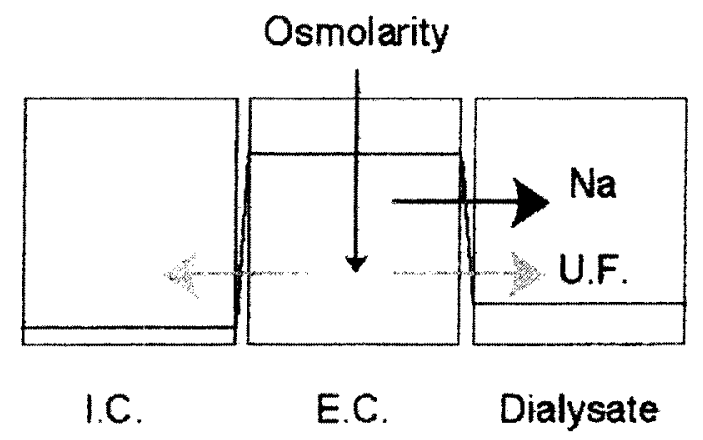

(1) Low Na Dialysate

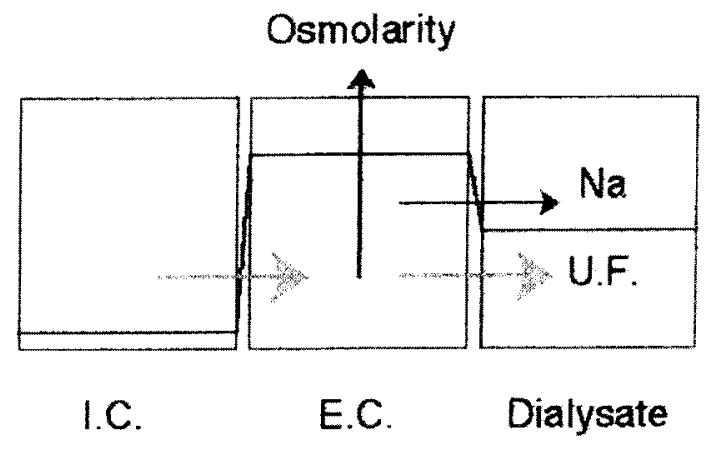

(2) High Na Dialysate

FIGURE 2 The interaction between sodium removal and water shifts during a haemodialysis session. (1) Low Na dialysate: a low dialysate sodium results in rapid removal of sodium. If the ultrafiltration rate is not high, the osmolarity of the extracellular compartment falls relative to that of the intracellular compartment and the dialysate. The net result is for water to move from the extracellular compartment to the dialysate and also into the intracellular compartment. (2) High sodium dialysate: the removal of sodium is reduced and, together with ultrafiltration, results in an increase in extracellular osmolarity, which in turn leads to removal of water from both the intracellular and extracellular compartments

maximum simplicity for our model, we will assume that the sum total of osmolarity due to all osmolytes other than sodium remains constant during the dialysis session. This approximation may not be applicable when considering poorly controlled diabetic patients.

(2) Disequilibrium syndrome is mainly due to overhydration of central neurones, resulting from intracellular influx of water. In practice, disequilibrium syndrome is likely to occur when the EC sodium concentration decreases by more than 7 $\mathrm{mmol} / \mathrm{l}$ during dialysis [5]. This would result in a significant reduction in plasma osmolarity and hence the movement of fluid from the EC compartment to the IC compartment.

The onset of symptomatic hypotension and disequilibrium syndrome can be limited by preventing a rapid reduction in plasma osmolarity. The two means of preventing such a fall in plasma osmolarity are (i) intradialytic injection of a sodium bolus or hypertonic solution (for example, to treat muscle cramps) and (ii) profiling of dialysate sodium. In (i) the change in osmolarity is direct and rapid, whereas in (ii), increasing the dialysate sodium reduces (or even reverses) the diffusion of sodium from the EC compartment into the dialysate and offsets any reduction in plasma osmolarity. Whereas these measures are often successful in preventing or alleviating intradialytic problems, they risk preventing adequate sodium removal during dialysis. This can lead to a viscious cycle in which increasing sodium levels encourage the patient to drink more, causing fluid overload, such that higher ultrafiltration rates are needed to achieve dry weight, risking hypovolaemia and the need for further hypertonic saline injection. Moreover, in a patient overloaded with fluid, the actual EC sodium mass is not reflected by the EC sodium concentration and so the dialysate sodium level may be underestimated: in a series of dialysis sessions, the patient's plasma sodium concentration may be stable, while they become increasingly fluid overloaded. Hypertension is a common and difficult problem in many patients, often related to volume overload, and is a major factor responsible for the high cardiovascular morbidity and mortality in dialysis patients. Thus in striving to prevent intradialytic morbidity by control-

* Such patients account for around a third of patients on dialysis. 
ling EC sodium, and therefore plasma osmolarity, we risk hypernatraemia, thirst, fluid overload and hypertension. An important challenge for profiled dialysis is to combine intradialytic plasma osmolarity control with adequate solute removal.

\section{TARGETS AND CONSTRAINTS OF PROFILED DIALYSIS}

The foregoing discussion highlights the complications that are often associated with changes in plasma osmolarity during haemodialysis. It is likely that longer dialysis times with lower ultrafiltration rates would reduce intradialytic events linked to changes in plasma osmolarity. However, the drawbacks of longer treatment times are the extra cost and patient acceptability. In the following, we outline some of the key requirements of successful dialysis treatment.

(1) Sufficient sodium mass should be removed to prevent the fluid overload cycle take oves (2) The ultrafiltration rate should be monotonic and not increase later, as patients usually tolerate higher rates at the start of a dialysis session. Some success has been found with linearly decreasing ultrafiltration rates.

(3) Fluctuations (particularly increases which may lead to disequilibrium syndrome) in ICV should be minimised.

(4) Rate of loss of ECV should be minimised to prevent symptomatic hypotension due to hypovolaemia.

(5) The EC sodium concentration should lie between $135.0 \mathrm{mmol} / \mathrm{l}$ and $145.0 \mathrm{mmol} / \mathrm{l}$.

(6) Adequate removal of urea and middle molecules.

\section{A SIMPLE MODEL FOR SODIUM PROFILING}

Our simple model (see Figure 3) develops along similar lines to that of [1] and [2] although owing to the relatively small contribution of urea removal to fluid shifts [5], [6], [9] we choose not to include urea kinetics. This simplification makes the model easier to solve, which we believe is not unreasonable for a practical model. However, the model cannot predict urea rebound [7], nor the transient effects due to rapid urea equilibration. As mentioned above, the IC mass of sodium is assumed to remain constant [item (3) page 4]. We introduce the following notation:

$M_{N a}^{e}(t)=\mathrm{EC}$ molar masses of sodium

$M_{N a}^{i}=$ IC molar masses of sodium, assumed constant

$M_{\text {other }}^{e}(t), \quad M_{\text {other }}^{i}(t)=\mathrm{EC}$, IC molar masses of other solutes

$V^{e}(t)=\mathrm{ECV}, V^{i}(t)=\mathrm{ICV}$

$V(t)=$ total body water volume

$C_{N a}^{e}(t)=$ EC concentration of sodium $=M_{N a}^{e}(t) / V^{e}(t)$

$C_{N a}^{i}(t)=\mathrm{IC}$ concentration of sodium $=$

$M_{N a}^{i}(t) / V^{i}(t)$

$C_{\text {other }}^{e}(t)=\mathrm{EC}$ concentration of other solutes = $M_{\text {other }}^{e}(t) / V^{e}(t)$

$C_{\text {other }}^{i}(t)=$ IC concentration of other solutes = $M_{\text {other }}^{i}(t) / V^{i}(t)$

$C_{N a}^{d}(t)=$ dialysate concentration of sodium

$k_{d}=$ dialyser flux constant for sodium

$k_{m}=$ cell membrane flux constant for sodium

$U(t)=$ ultrafiltration rate, as controlled by hydraulic pressure across the dialyser membrane

$B(t)=$ blood flow rate at the dialyser inlet, as contolled by the quality of the blood access and blood pump rate.

First we deal with the mass balance equations which describe the fluxes of each solute through the IC and EC fluid compartments and across the dialyser membrane. Neglecting sodium ingestion, the equation 
for the rate of change of mass of sodium in the EC fluid is, in words,

$$
\underbrace{\begin{array}{c}
\text { rate of change of } \\
\text { EC sodium mass } \\
\begin{array}{c}
\text { IC EC sodium } \\
\text { flux due to passive } \\
\text { diffusion across } \\
\text { cell membrane }
\end{array}
\end{array}}_{2}+\underbrace{\begin{array}{c}
\text { IC } \rightarrow \text { EC sodium } \\
\text { pumping of sodium } \\
\text { across cell membrane }
\end{array}}_{3}
$$

Let us look at the terms of equation (1) individually. Term 1 is simply the time derivative of the EC sodium mass, $d M_{N a}^{e}(t) / d t$. Term 2 is the net mass of sodium diffusing from the IC compartment per unit time. From Fick's law, this is proportional to the IC:EC concentration difference. If the constant of proportional is $k_{m}$, the second term is $k_{m}\left(C_{N a}^{i}(t)-C_{N a}^{e}(t)\right)$. Thus if the IC sodium concentration exceeds that of the EC compartment, there is a positive flux into the $\mathrm{EC}$ compartment, and the larger the membrane permeability $k_{m}$, the higher the flux. Term 3 is the mass of sodium pumped from the IC compartment into the EC compartment by ATP-driven sodium pumps. Term 4 is similar to term 2 , except now the transfer is from the EC compartment into the dialysate. Term 5 accounts for the solute that is transported from the EC compartment into the dialyser due to ultrafiltration. In this case solute is carried with the ultrafiltrate at a rate $U(t) C_{N a}^{e}(t)$. Thus increasing the ultrafiltration not only increases the rate of removal of water, but also the convective transport of solutes. Note that this convectional flux does not change the concentration of the EC compartment: only passive diffusion of solute can achieve this. Putting all these terms together we obtain:

$$
\begin{aligned}
\underbrace{\frac{d M_{N a}^{e}(t)}{d t}}_{1} & =\underbrace{k_{m}\left(C_{N a}^{i}(t)-C_{N a}^{e}(t)\right)}_{2}+\underbrace{J_{N a}^{p u m p}}_{3} \\
& +\underbrace{D_{N a}\left(1-\frac{U(t)}{B}\right)\left(C_{N a}^{d}(t)-C_{N a}^{e}(t)\right)}_{4} \\
& +\underbrace{U(t) C_{N a}^{e}(t)}_{5}
\end{aligned}
$$

Here $D_{N a}$ is the dialysance of the dialyser to sodium, defined to be the change in solute content of the blood entering the dialyser per concentration driving force:

$$
D_{N a}=\frac{B\left(C_{N a}^{\text {in }}(t)-C_{N a}^{o u t}(t)\right)}{\left(C_{N a}^{i n}(t)-C_{N a}^{d}(t)\right)}
$$

At constant blood flow rates, this is a constant for the dialyser. $C_{N a}^{i n}(t)\left(C_{N a}^{o u t}(t)\right)$ is the sodium concentration of the blood entering (leaving) the dialyser. For more details on how to derive terms 4 and 5 see [11]. They can be understood as follows by considering two extreme operating conditions. When the blood inflow rate $B$ equals the ultrafiltration rate $U$ there is no diffusional contribution to the dialyser flux (i.e. term 4 in equation (2) vanishes), and the entire flux is convectional (term 5 in equation (2)). Conversely, when there is no ultrafiltration, so that $U(t)=0$, all of the flux is diffusional. Now let $\alpha(t)=(1-U(t) / B(t))$ and suppose constant rates $U(t)$ $=1.0 \mathrm{l} / \mathrm{h}$ and $B(t)=0.3 \mathrm{l} / \mathrm{min}$ are chosen (as in [2]). Then the correction factor

$$
\alpha=\left(1-\frac{1}{60 \times 0.3}\right)=0.95
$$

Hence under such conditions we may reasonably approximate the correction factor as $\alpha=1$. Assuming a fixed and constant blood flow rate $B(t)=0.3 \mathrm{l} / \mathrm{min}$, this approximation still holds good even with time variations of the ultrafiltration rate in the range of $\pm 0.5 \mathrm{l} / \mathrm{h}$ around a mean value of $1.0 \mathrm{l} / \mathrm{h}$. To simplify matters, we will therefore ignore this correction factor. 


$$
\begin{aligned}
\underbrace{\frac{d M_{N a}^{e}(t)}{d t}=}_{1} & \underbrace{k_{m}\left(C_{N a}^{i}(t)-C_{N a}^{e}(t)\right)}_{2}+\underbrace{J_{N a}^{p u m p}}_{3} \\
& +\underbrace{D_{N a}\left(C_{N a}^{d}(t)-C_{N a}^{e}(t)\right)}_{4} \\
& +\underbrace{U(t) C_{N a}^{e}(t)}_{5}
\end{aligned}
$$

Recall that one of our assumptions has been that the IC sodium mass remains constant. Suppose that, during a particular dialysis session, water and sodium are being lost to the dialysate by diffusion and ultrafiltration in such a way that the EC volume reduces more rapidly than the EC sodium mass, so that the EC sodium concentration rises. This will result in a IC:EC sodium gradient that favours more net transport of sodium into the IC compartment. Typically, this would be countered by increased activity of the sodium pumps, so as to return the sodium to the EC compartment. The ability of the cells to maintain their internal sodium mass constant thus depends on them being able to harness sufficient sodium pumping when required. Our assumption that the IC mass is constant thus translates into the sodium pump flux balancing the net passive flux of sodium into the cell, and thus terms 2 and 3 cancel. Mathematically, this simplifies equation (4) to

$$
\begin{aligned}
\underbrace{\frac{d M_{N a}^{e}(t)}{d t}}_{1} & =\underbrace{D_{N a}\left(C_{N a}^{d}(t)-C_{N a}^{e}(t)\right)}_{4} \\
& -\underbrace{U(t) C_{N a}^{e}(t)}_{5} \\
= & -\left(D_{N a}+U(t)\right) C_{N a}^{e}(t)+D_{N a} C_{N a}^{d}(t)
\end{aligned}
$$

Now let us turn to the volume changes, the total body water volume is the sum of the IC and EC volumes:

$$
V(t)=V^{i}(t)+V^{e}(t)
$$

The rate of removal of water by ultrafiltration is

$$
\frac{d V(t)}{d t}=-U(t)
$$

so that by integration, the total volume at a given time $t$ is

$$
V(t)=V(0)-\int_{0}^{t} U(t) d t
$$

In most dialysis sessions, $U(t)$ will be positive, so that the volume reduces with time. When the ultrafiltration is constant, say $U(t)=U_{0}$ this last equation reduces to

$$
V(t)=V(0)-U_{0} t
$$

and corresponds to a linearly decreasing total body volume.

We now come to the volume changes in each of the IC and EC compartments. These are the result of both ultrafiltration and osmosis. Since the fluid is incompressible, we may assume that volume changes are in constant proportion to water mass changes. For the $\mathrm{EC}$ volume changes, we have in words:

$$
\begin{aligned}
& \text { rate of change }= \\
& \underbrace{\text { of ECV }}_{1}= \\
& \mathrm{IC} \rightarrow \mathrm{EC} \text { water flux } \quad \mathrm{EC} \rightarrow \text { dialysate } \\
& + \text { due to osmotic - ultrafiltrational } \\
& \underbrace{\text { pressure gradient }}_{2} \underbrace{\text { water flux }}_{3}
\end{aligned}
$$

As before, we will deal with each term individually. The first term is the time derivative of the ECV $d V^{e}(t) / d t$. As mentioned above this is proportional to the rate of change of EC water mass. Term 2 is the mass of water per unit time flowing from the IC compartment into the $\mathrm{EC}$ compartment and is proportional to the osmotic pressure difference $\Delta \pi$ with constant of proportionality is $k_{0}$. The difference $\Delta \pi$ is positive when the osmolarity of the IC and EC solutions is such that water moves from the IC to the EC compartment. Term 3 accounts for the loss of EC fluid to the dialysate due to ultrafiltration. Similarly, for the IC volume changes, we have

$$
\begin{gathered}
\text { rate of change } \\
\text { of ICV }
\end{gathered}=\begin{gathered}
\mathrm{IC} \rightarrow \mathrm{EC} \text { water flux } \\
\text { due to osmotic } \\
\text { pressure gradient }
\end{gathered}
$$

Thus the equations for the EC and IC fluid volume changes are 


$$
\begin{array}{r}
\frac{d V^{e}(t)}{d t}=k_{0} \Delta \pi(t)-U(t) \\
\frac{d V^{i}(t)}{d t}=-k_{0} \Delta \pi(t)
\end{array}
$$

The presence of water permeable channels in the typical cell membrane makes it relatively permeable to water, which means that $k_{0}$ is large. Notice that adding these two equations and using $V(t)=V^{e}(t)+V^{i}(t)$, we regain equation (7).

To complete the description of the volume changes, we need a formula for the osmotic pressure gradient between the IC and EC compartments. The osmotic pressure difference between the IC and EC fluid compartments is like any other concentration difference in that it is proportional to the difference in water concentrations between the two compartments. Where there is a solute particle it displaces a water molecule, and so the water concentration of each compartment is determined by the number of solute particles in the compartment. The osmotic pressure difference is thus proportional to the difference in the number of solute particles between the IC and EC compartments. This is summarised by the van't Hoff formula (e.g. [9]):

IC $\rightarrow$ EC transmembrane

osmotic pressure difference $\propto$

number of solute number of solute

particles in $\mathrm{ICV}-$ particles in $\mathrm{ECV}$

or in equation form

$$
\Delta \pi=R T\left(\sum_{\text {solutes } k} C_{k}^{i}(t)-\sum_{\text {solutes } k} C_{k}^{e}(t)\right)
$$

The constants $R, T$ in the above formula are the universal gas constant and the absolute temperature, respectively. There are a large number of different solutes to consider in each cell. Outside a dialysis session, we may assume that these solutes are distributed in order to establish osmotic balance (i.e. $\Delta \pi=0$ ). During dialysis, however, the removal of water and solutes disturbs the concentrations of some key solutes in each compartment and this results in an osmotic gradient. Solutes that can permeate the membrane freely, such as potassium, and for some cell or tissue types, glucose, will not contribute much to the osmotic pressure since any IC:EC differences are rapidly cancelled by diffusion. As the cell membrane is effectively impermeable to sodium, this solute and its partner anion, such as chloride or bicarbonate, will make the main contribution to the osmotic pressure. A second solute of importance is urea, since it is present at a much higher concentration in a dialysis patient. The cell membrane is relatively permeable to urea, so that its contribution to the osmotic gradient is considerably less than that of sodium. We group the solutes into sodium and urea, and a subgroup of 'other' solutes which are not in equal concentrations in the IC and EC compartments, but whose compartmental masses do not change during a dialysis session. These will include, for example, potassium, the dominant intracellular cation, chloride ions and the large negatively charged IC proteins which cannot permeate the cell membrane [9]. All the remaining solutes will not significantly contribute to the osmotic pressure. Then we obtain:

$$
\begin{aligned}
& \Delta \pi(t)= \\
& R T\left(\left\{2 C_{N a}^{i}(t)+C_{\text {urea }}^{i}(t)+\sum_{\text {others }} C_{k}^{i}(t)\right\}\right. \\
& \left.-\left\{2 C_{N a}^{e}(t)+C_{\text {urea }}^{e}(t)+\sum_{\text {others }} C_{k}^{e}(t)\right\}\right)
\end{aligned}
$$

The factor of 2 preceding each sodium concentration is to account for the fact that sodium is present in ionised form with its counterion. In terms of the molar masses this equation can be rewritten as

$$
\begin{aligned}
& \Delta \pi(t)= \\
& R T\left(\frac{2 M_{N a}^{i}(t)+M_{\text {urea }}^{i}(t)+\sum_{\text {others }} M_{k}^{i}}{V^{i}(t)}\right. \\
& \left.-\frac{2 M_{N a}^{e}(t)+M_{\text {urea }}^{e}(t)+\sum_{\text {others }} M_{k}^{e}}{V^{e}(t)}\right)
\end{aligned}
$$

In this paper, we will ignore the contribution of urea to the osmotic pressure, because the membrane is highly permeable to urea, and removal of urea from the EC volume to the dialyser is likely to create only a small IC:EC difference in urea concentration. Furthermore the size of this difference will become progressively smaller during the dialysis session as urea 
is removed and the compartmental urea concentration falls. Furthermore, since the cell membrane is highly permeable to water, we will assume that, to a first approximation, the movement of water from a compartment of low osmolarity to one of higher osmolarity is sufficiently rapid that, on the time scale of solute removal in a dialysis session, the IC and EC compartments are in osmotic balance.

With these assumptions, we may establish the following osmotic balance condition from equation (16) that imposes a constraint on the IC and EC sodium concentrations. Letting

$M_{\text {other }}^{e}=\sum_{\text {others }} M_{k}^{e}$ and $M_{\text {other }}^{i}=\sum_{\text {others }} M_{k}^{i}$

we have:

$$
\frac{2 M_{N a}^{i}+M_{o t h e r}^{i}}{V^{i}(t)}=\frac{2 M_{N a}^{e}(t)+M_{o t h e r}^{e}}{V^{e}(t)}
$$

We rewrite this equation to express the ECV in terms of the sodium mass and the total body water volume. From equation (6), $V^{i}(t)=V(t)-V^{e}(t)$, and so substituting this relation into equation (17) we obtain

$$
\frac{2 M_{N a}^{i}+M_{o t h e r}^{i}}{V(t)-V^{e}(t)}=\frac{2 M_{N a}^{e}(t)+M_{o t h e r}^{e}}{V^{e}(t)}
$$

Let

$M_{0}=M_{\text {other }}^{e}$ and $M_{1}=M_{\text {other }}^{e}+M_{\text {other }}^{i}+2 M_{N a}^{i}$.

Then equation (18) can be rearranged to give

$$
V^{e}(t)=\frac{V(t)\left(2 M_{N a}^{e}(t)+M_{0}\right)}{2 M_{N a}^{\epsilon}(t)+M_{1}}
$$

Now we are able to write down an expression for the EC sodium concentration by rearranging (19):

$$
C_{N a}^{e}(t)=\frac{M_{N a}^{e}(t)}{V^{e}(t)}=\frac{M_{N a}^{e}(t)\left(2 M_{N a}^{e}(t)+M_{1}\right)}{V(t)\left(2 M_{N a}^{e}(t)+M_{0}\right)}
$$

Finally, we may use this expression for the EC sodium concentration in the differential equation for the rate of change of EC sodium mass [equation (5)]:

$$
\begin{aligned}
& \frac{d M_{N a}^{e}(t)}{d t}= \\
& -\left(D_{N a}+U(t)\right) \frac{M_{N a}^{e}(t)\left(2 M_{N a}^{e}(t)+M_{1}\right)}{V(t)\left(2 M_{N a}^{e}(t)+M_{0}\right)} \\
& +D_{N a} C_{N a}^{d}(t)
\end{aligned}
$$

In the practical setting, the clinician is free to choose the ultrafiltration rate $U(t)$ and the dialysate sodium concentration $C_{N a}^{d}(t)$ as functions of time. In standard dialysis, the sodium dialysate level is generally kept constant, whereas the ultrafiltration rate is either constant or is set as a linearly decreasing function of time [5]. By contrast, during a profiled dialysis session, one, or both, the ultrafiltration rate and the sodium dialysate concentration may be defined as functions of time.

\section{A PRELIMINARY TEST OF THE MODEL}

At present the exercise is to test the validity of the model and to check that its predictions are consistent with published clinical observations. In future, we plan to fit the model with new and more detailed data from an operational dialysis unit (see Discussion).

In the absense of detailed data of our own, we have first compared data taken from Mann and Stiller's 1996 paper [5] with predictions of our model. In [5] (Figure 8) the authors show the variation of blood sodium concentration for a five hour profiled haemodialysis session where the dialysate sodium profile is hourly stepped between $140 \mathrm{mmol} / \mathrm{l}$ and $160 \mathrm{mmol} / \mathrm{l}$. The resulting blood sodium concentration increases from $140 \mathrm{mmol} / \mathrm{l}$ to $152 \mathrm{mmol} / \mathrm{l}$. We have used these data to test our model. Since there is zero ultrafiltration, the total body water volume remains constant. Changes in EC sodium concentration are then calculated from equation (21) with $U(t) \equiv 0$ and $V(0)=V_{0}$. Next the EC volume profile $V^{e}(t)$ is found using (19) and finally the IC volume from $V^{i}(t)=V(t)-V^{e}(t)=V_{0}-V^{e}(t)$. The patient parameters we used, listed in Table $I$, were taken from the published literature and apply to a patient with a dry weight of $65 \mathrm{~kg}$. The only parameter missing is $M_{\text {other }}^{i}=C_{\text {other }}^{i}(0) / V^{i}(0)$. Asssuming osmotic equilibrium initially,

$$
2 C_{N a}^{i}+C_{\text {other }}^{i}(0)=2 C_{N a}^{e}(0)+C_{\text {other }}^{e}(0)
$$

we find

$$
M_{\text {other }}^{i}=\left(2 C_{N a}^{e}(0)+C_{\text {other }}^{e}(0)-C_{N a}^{i}(0)\right) / 2 V^{i}(0) \text {. }
$$




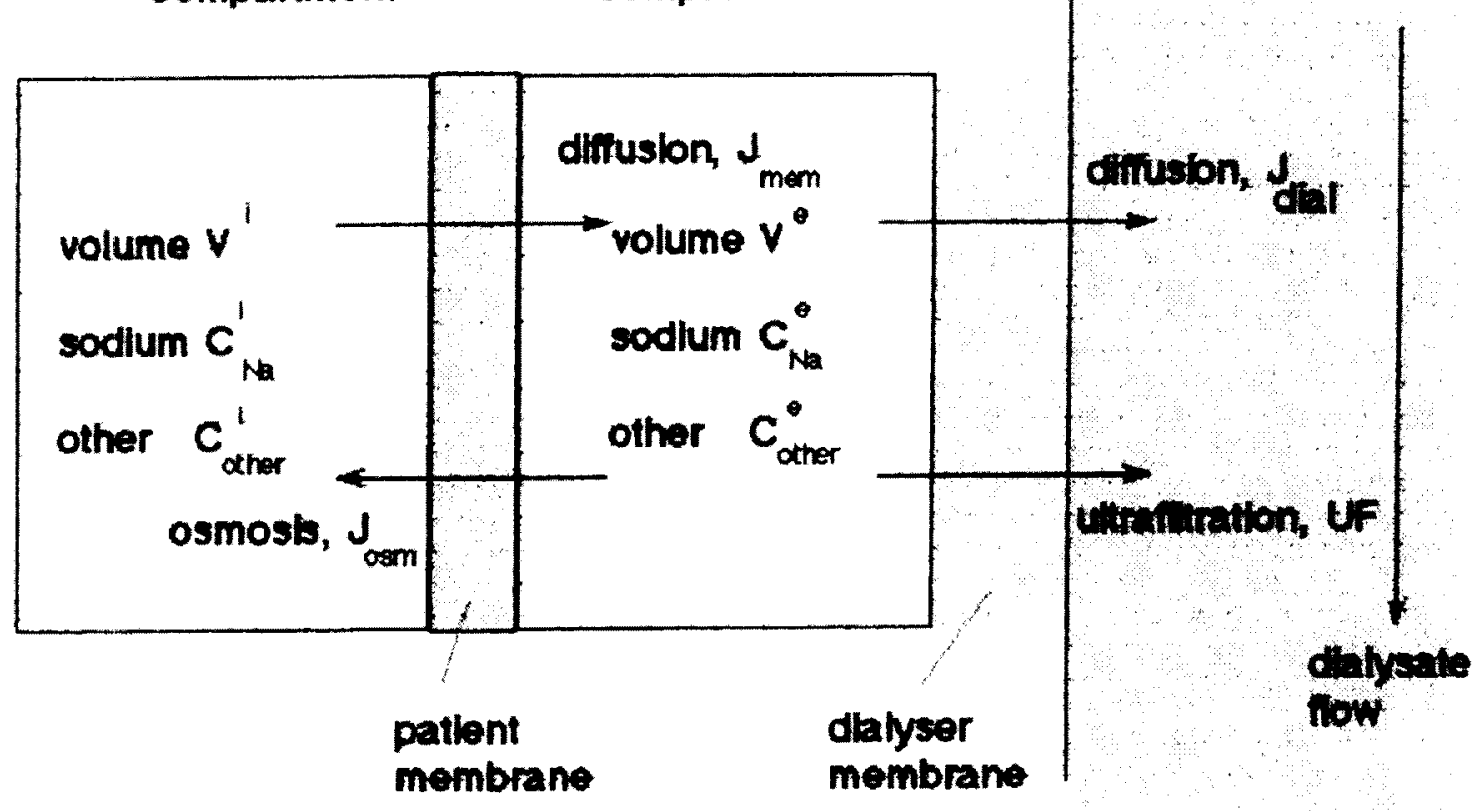

FIGURE 3 Summary of the variable-volume, two-compartment model for the haemodialysis process. The patient's body fluids are partitioned into two compartments, the intracellular and extracellular compartments, separated by a biological membrane representing the various cell membranes and interstitial space across which solutes and solvent must diffuse. The transport of osmotically active sodium across this barrier leads to fluid osmotic flux, $J_{\mathrm{osm}}$. The extracellular compartment is separated from the dialysis machine by an artificial membrane through which solute and solvent can be both passively and convectively transported

To obtain the actual masses of sodium in grams we multiply the molar masses by the molecular weight factor 23. In line with much of the published data, we will identify the mass of sodium removed with the mass of $\mathrm{NaCl}$ (molecular weight 58.5) removed. Figure 4 shows our model simulation carried out with zero ultrafiltration and an identical dialysate sodium profile to that described in [5]. Running from top to bottom in the left column the curves show profiles of dialysate sodium concentration, EC sodium concentration in $\mathrm{mmol} / \mathrm{l}$ and $\mathrm{EC}$ volume in litres. In the right column, again from top to bottom we plot ultrafiltration rate in litres/hour, EC sodium mass (i.e. mass of $\mathrm{NaCl}$ ) in grams and IC volume in litres. We note that there is broad agreement with [5]; in particular we predict an increase in EC sodium concentration from $140 \mathrm{mmol} / /$ to $152 \mathrm{mmol} / \mathrm{l}$. Furthermore, we predict the net removal of sodium to be $22.6 \mathrm{~g}$ against their value of $25 \mathrm{~g}$.

TABLE I Typical patient parameters used to fit the model. Parameters were taken from [2]

\begin{tabular}{cc}
$V(0)$ & 37.7 litres \\
$C_{N a}^{e}(0)$ & $145.0 \mathrm{mmol} / \mathrm{litre}$ \\
$C_{N a}^{i}$ & $5.0 \mathrm{mmol} / \mathrm{itre}$ \\
$C_{o t h e r}^{e}(0)$ & $90.0 \mathrm{mmol} / \mathrm{litre}$ \\
$k_{d}$ & 12.84 litre/hour \\
\hline
\end{tabular}



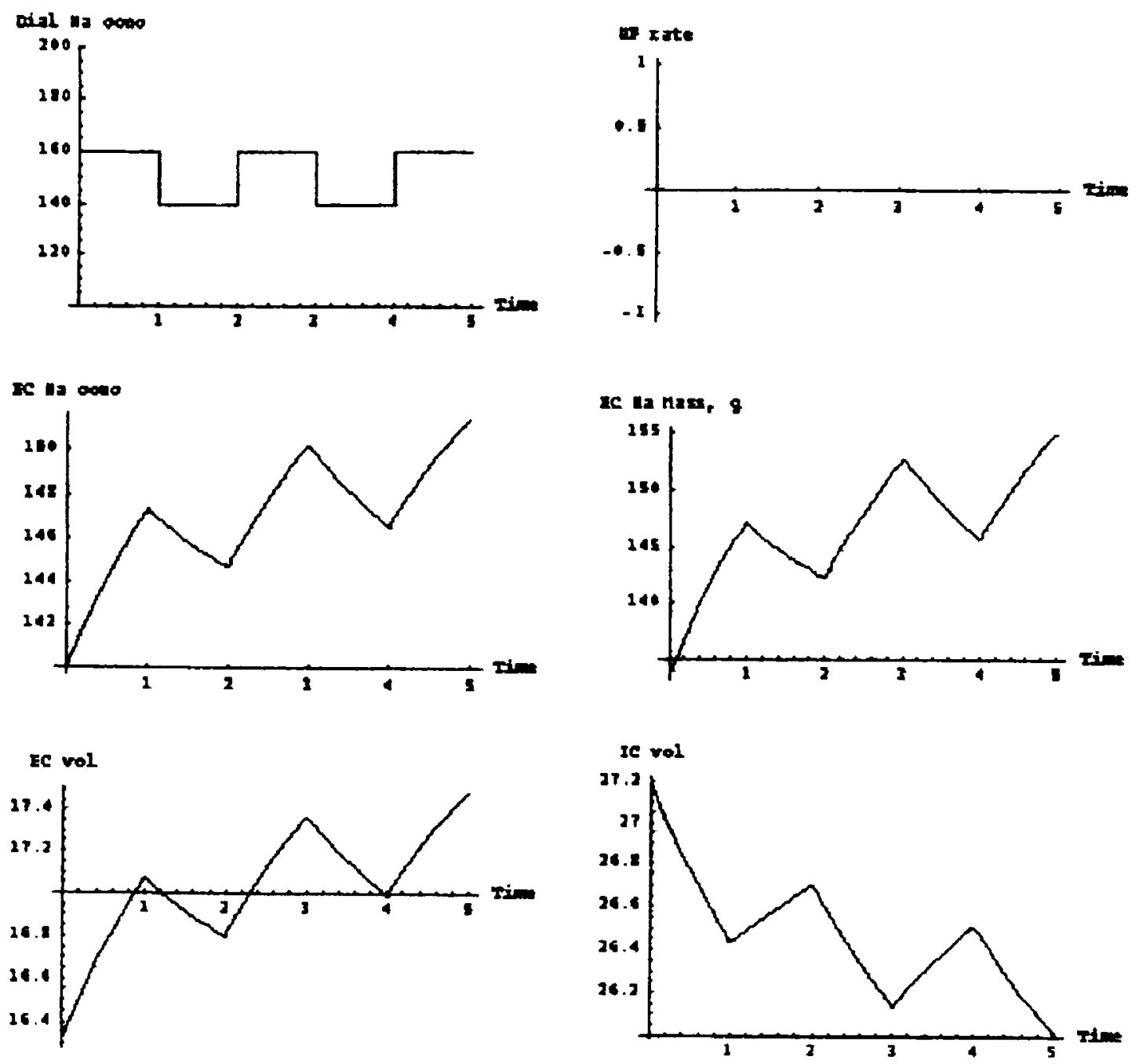

FIGURE 4 Test of the model against the data of Mann and Stiller [5] for a step wise sodium profile over a five hour dialysis session, with zero ultrafiltration. The model predicts a $22.6 \mathrm{~g}$ net gain of sodium, compared with $25 \mathrm{~g}$ quoted by Mann and Stiller

\section{OPTIMISING PROFILED DIALYSIS}

Since control of the ECV is a specific aim, so as to prevent a fall in EC osmolarity, we would like to find the sodium dialysate concentration profile which generates a given ECV profile, and, if possible, to also achieve targets for fluid and sodium removal. This can be done as follows. First we show how to calculate the dialysate sodium profile once the ultrafiltration and ECV profiles are set. The key is equation (20) rearranged in terms of the sodium dialysate concentration $C_{N a}^{d}(t)$ : 


$$
\begin{aligned}
& C_{N a}^{d}(t)= \\
& \frac{1}{D_{N a}} \frac{d M_{N a}^{e}(t)}{d t} \\
& +\left(1+\frac{U(t)}{D_{N a}}\right)\left(\frac{M_{N a}^{e}(t)\left(2 M_{N a}^{e}(t)+M_{1}\right)}{V(t)\left(2 M_{N a}^{e}(t)+M_{0}\right)}\right)
\end{aligned}
$$

Now, once $U(t)$ is known so is $V(t)$ by equation (8). If $V^{e}(t)$ is also given, then we may calculate $M_{N a}^{e}(t)$ by rearranging equation (19) in terms of $V^{e}(t)$. Finally we substitute the calculated profile for the EC sodium mass $M_{N a}^{\epsilon}(t)$ into (23) to find the necessary sodium dialysate profile. This must be done while removing specified quantities of fluid and sodium. Thus we now demonstrate how to remove a specified amount of fluid. We consider only constant and linearly decreasing ultrafiltration rates for a dialysis session running from $t=0$ to $t=T$, and for which the profile $V^{e}(t)$ is set. The total fluid volume removed at time $T$ is $\Delta V(T)$ where

$$
\Delta V(T)=V(T)-V(0)=-\int_{0}^{T} U(T) d t
$$

The number $\Delta V(T)$ is prescribed as the amount of fluid to be removed. When $U(t)=U$, a constant, the constant ultrafiltration required to remove $\Delta V(T)$ in time $T$ is $U=\Delta V(T) / T$. Next we have to ensure that a given net mass of sodium is removed. Let $\Delta M_{N a}^{e}=M_{N a}^{e}(0)-M_{N a}^{e}(T)$ be the amount of sodium to be removed during the dialysis session. From $M_{N a}^{e}(T)=M_{N a}^{e}(0)-\Delta M_{N a}^{e}$ and equation (19) with $t=T$ we have

$$
V^{e}(T)=V(T)\left(\frac{2 M_{N a}^{e}(0)-2 \Delta M_{N a}^{e}+M_{0}}{2 M_{N a}^{e}(0)-2 \Delta M_{N a}^{e}+M_{1}}\right)
$$

All the parameters and constants on the right-hand side of equation (25) are known, so that the final ECV of $V^{e}(T)$, must satisfy equation (25) for the target of sodium mass and fluid removal to be achieved. To summarise, we first decide upon an ultrafiltration rate that removes the correct amount of fluid. Then we select an 'optimal' ECV profile $V^{e}(t)$ chosen from all those profiles that satisfy the same end conditions $V^{e}(0)=V_{0}^{e}$ and $V^{e}(T)=V_{T}^{e}$.

The question now arises: how do we specify an 'optimal' ECV profile? As mentioned above, the final
$\mathrm{ECV}$ is fixed once the ultrafiltration rate and the amount of sodium to be removed have been decided upon. What is at our disposal is choosing how ECV should vary between its start and end values. We thus generated ECV profiles using the functional form

$$
V^{e}(t)=V_{0}^{e}+b t+a t^{2}
$$

where $b=\left(V^{e}(T)-V^{e}(0)-a T^{2}\right) / T$ and $a$ was chosen to yield three distinctly shaped profiles: (i) $a=0$ (linear), (ii) $a=-0.15$ (concave), and (iii) $a=0.15$ (convex). In [22] the authors use a similar approach in which they seek to optimise the dialysis session by iterating the EC sodium mass profile to maintain high EC sodium mass in the first half of dialysis. This method also allows the direct computation of the dialysate sodium profile required to realise the optimal session. Due to the manner in which EC sodium and ECV are coupled in our model (equation (18)), and since we are assuming constant ultrafiltration, we can mimic maintaining high EC sodium mass in the first half of the session by chosing a similar profile for the ECV (see Figure 8).

\section{Results}

Our results for the various profiles are shown in Figures 6 to 9 . For each of these figures the following general observations apply, and can be explained by the simple model. First, as mentioned already, in each case the final ECV is the same, regardless of the particular dialysate sodium profile: the final ECV is determined by the amount of sodium and fluid removed. Second, note that since the net quantity of fluid removed is the same in all cases, the final ICV is also identical.

Figure 5 shows that a linearly decreasing ECV can be achieved from a near constant dialysate sodium profile. In this case, the dialysis session succeeds in achieving the target sodium and fluid removal and is not likely to produce intradialytic morbidity. In Figure 6, however, we see that increasing the sodium removal target from $20 \mathrm{~g}$ to $30 \mathrm{~g}$ is likely to push $\mathrm{EC}$ sodium concentration too low. In Figure 7, we remove ECV more rapidly in the earlier stages of dialysis. Effectively, this is achieved by beginning with a dia- 

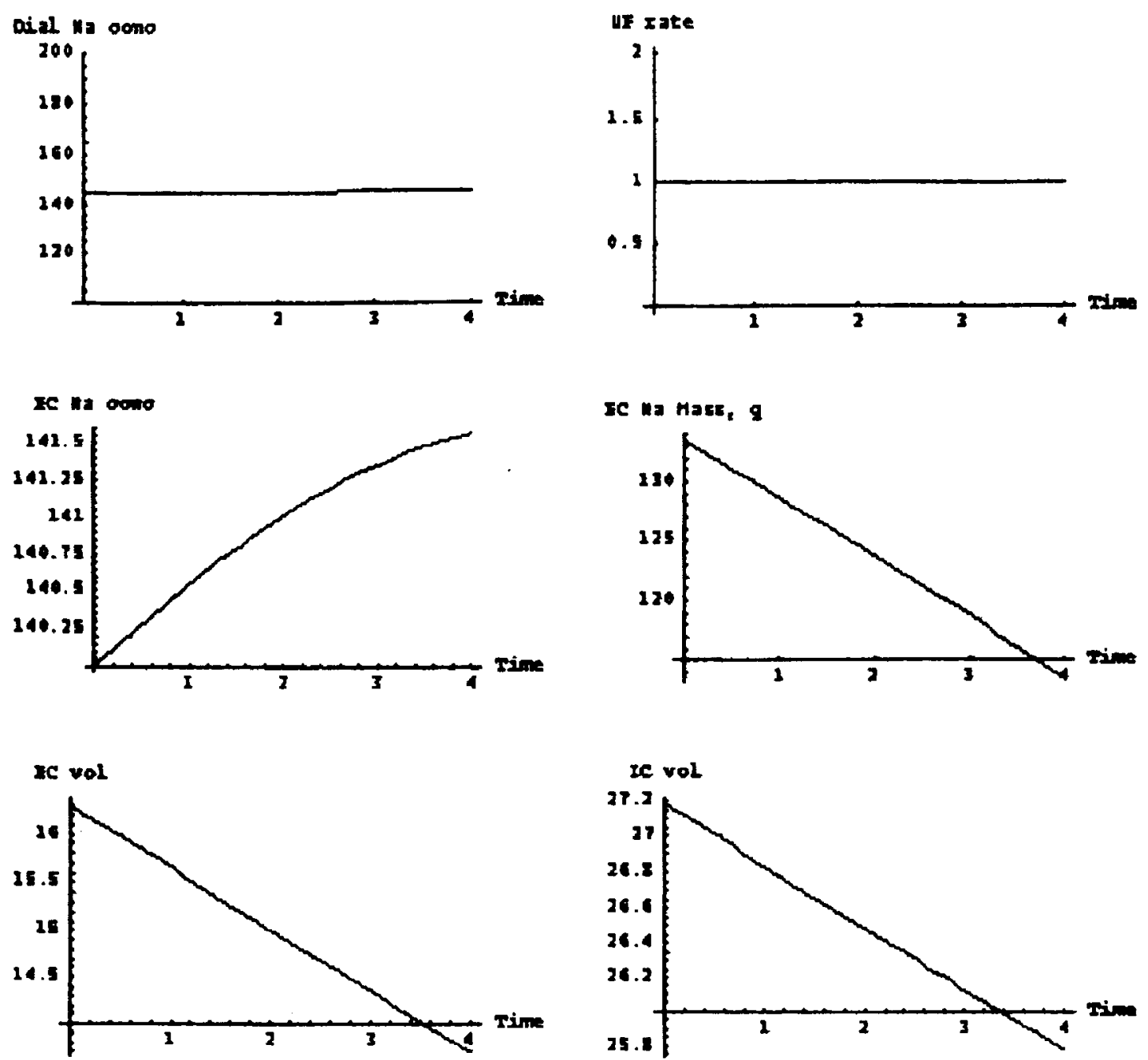

FIGURE 5 Simulation of profiled dialysis session (I). The ECV profile is designed to simultaneously remove 4 litres of fluid and $20 \mathrm{~g}$ of sodium. A linear ECV profile requires an approximately constant dialysate sodium profile. In this case reductions in sodium mass and ECV and ICV are also approximately linear

lysate sodium concentration below the EC sodium concentration and gradually increasing it to a level above the dialysate sodium concentration. As a result, EC sodium is rapidly removed early in the session, thus lowering EC osmolarity, and causing an initial increase in ICV. Later water returns from the ICV to the $\mathrm{ECV}$ as the removal EC sodium diminishes, but ultrafiltration remains constant, thus increasing EC osmolarity. In the last simulation, Figure 8, the initially high sodium results in an increase in EC sodium, which raises EC osmolarity and causes rapid flow of water from the ICV to ECV. This vascular refilling process prevents a rapid depletion in the ECV and is the basis of sodium profiling. As the sodium dialysate concentration is reduced, eventually more sodium is removed more extensively from the EC compartment and the EC osmolarity begins to fall, so that the ECV is reduced more rapidly, and eventually the IC volume experiences a small increase. 

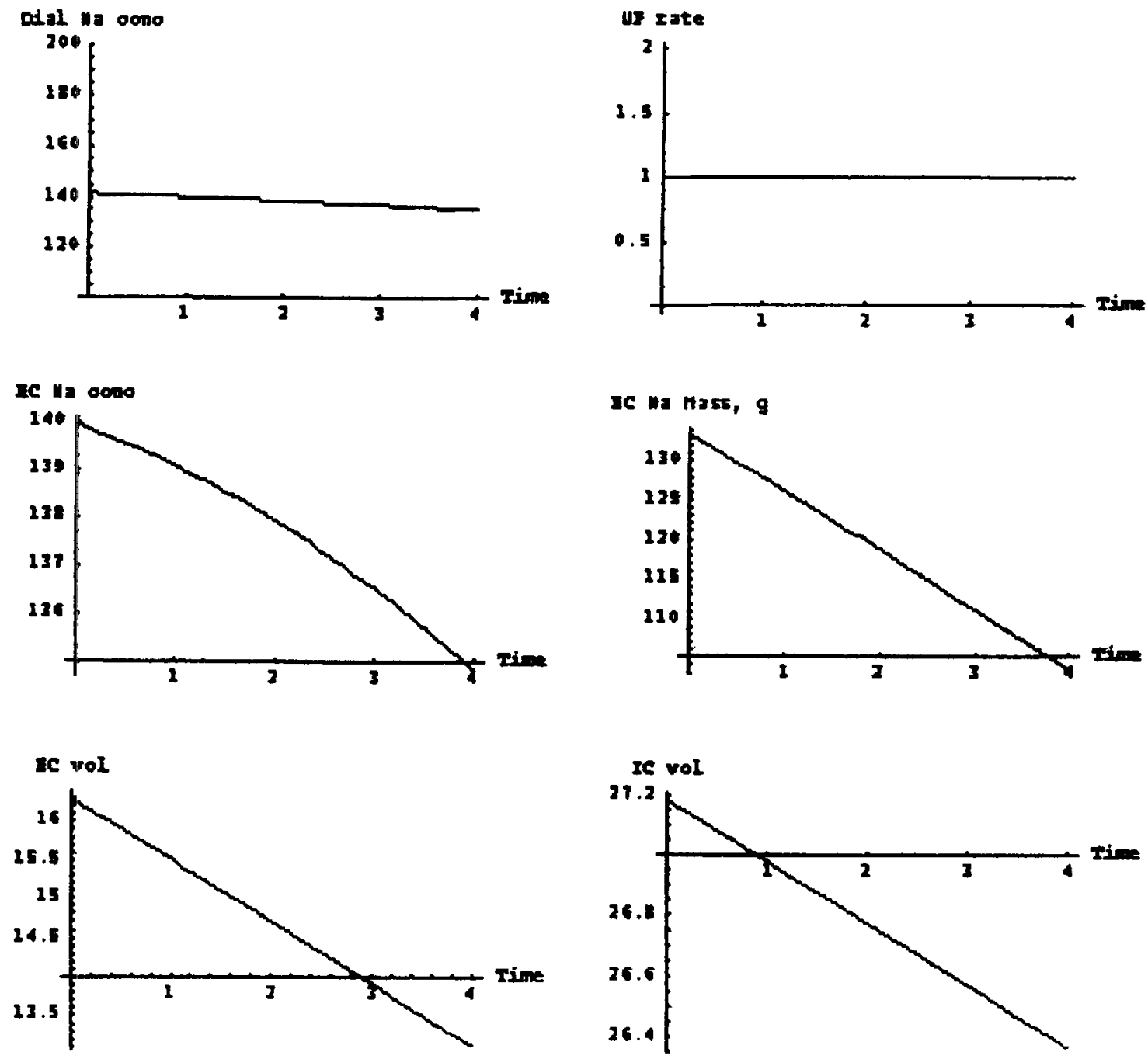

FIGURE 6 The same linear ECV profile as in Figure 5, except now the target for sodium mass removal is $30 \mathrm{~g}$. While the reductions in sodium mass and ICV and ECV remain linear, the EC sodium concentration falls significantly

\section{IDENTIFICATION OF 'SAFE' DIALYSIS REGIMES}

Using the simplified model we may suggest dialysis session settings that ensure that important patient variables remain within acceptable limits whilst achieving dialysis targets. We select the total fluid to be removed $\Delta V$ and total sodium mass to be removed $\Delta M_{N a}$ as parameters for a parameter space, and shade regions of parameter space where EC sodium concentration and ECV remain between pre-defined 'safe' limits throughout the simulated dialysis session. For the patient parameters listed in Table I the limits chosen are 

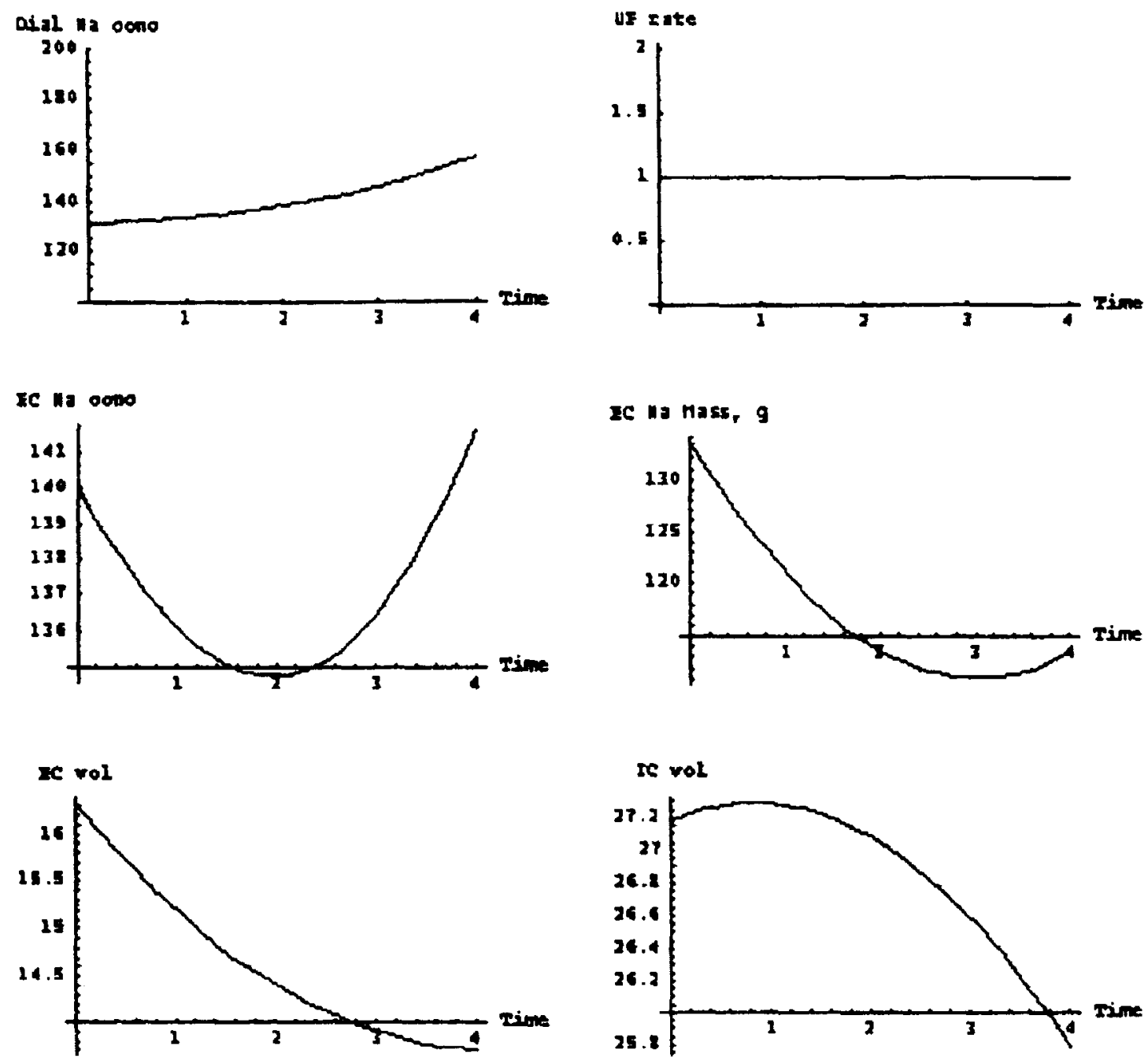

FIGURE 7 Simulation of profiled dialysis session (II). The ECV profile is designed to simultaneously remove 4 litres of fluid and $20 \mathrm{~g}$ of sodium. For this convex ECV profile, ECV reduces more rapidly in the earlier stages of dialysis. This is achieved by starting the dialysis session with a dialysate sodium concentration below the EC sodium concentration and gradually increasing it to a level above the dialysate sodium concentration. As a result, EC sodium is rapidly removed early in the session, thus lowering EC osmolarity, and causing an initial increase in ICV. Later water returns from the ICV to the ECV as the removal EC sodium diminishes, but ultrafiltration remains constant, thus increasing EC. osmolarity

$135 \mathrm{mmol} /$ litre $\leq C_{N a}^{e}(t) \leq 145 \mathrm{mmol} /$ litre, and $V^{e}(t) \geq 14.0$ litres

The results are shown in Figure 9, which shows three plots for a patient with dry weight $75 \mathrm{~kg}$, one for each of the ECV profiles (i) $a=0$ (linear), (ii) $a=-0.15$ (concave), and (iii) $a=0.15$ (convex). The plots suggest that a linear ECV profile is more likely to ensure that the patient's EC sodium concentration and ECV remain within acceptable limits. For non-linear ECV profiles, there is greater variation in the EC sodium concentration and this is a potential limitation of 
sodium profiling, which could be improved by simultaneously profiling the ultrafiltration. Taken together, these plots suggest that dialysate sodium should be profiled in order to achieve a linear decrease in ECV.

\section{DISCUSSION}

The purpose of this paper has been to introduce, in relatively simple terms, a bicompartmental model for a typical profiled haemodialysis session that would provide the clinician with practical criteria for setting suitable sodium profiles. The model has been tested for consistency with a sample of data and observations already reported in the literature. The detailed numerics of our simulations are therefore of limited relevance until the model has been fitted to data from patients undergoing dialysis. Nevertheless, the numerics enable us to explore the potential benefits of various profiled dialysis regimens, at least at the qualitative level; the chosen parameters ensure that the relative importance of transport terms remains consistent with clinical observations. To validate the model more comprehensively, it will be necessary to compare it against regular records of plasma sodium concentration, and intra- and extracellular fluid volumes taken from a wide range of standard and profiled dialysis sessions. There are a number of methods for measuring plasma sodium concentration; blood samples can be extracted non-invasively from the arterial line, or, as for some machines, the concentration can be continuously monitored with ion-sensitive electrodes. The simplest method for estimating the variation in total body fluids is to weigh the patient at regular intervals. The total body fluid content in litres is then estimated to be $58 \%$ of the body mass in kilograms. However, this does not provide estimates for the relative volumes of the ICV and ECV. More sophisticated, yet still indirect, approaches to measuring the distribution of body fluids are available. One technique [14] relies on the presence of a blood component $\mathrm{X}$ whose mass remains substantially constant during dialysis. Changes in $\mathrm{X}$ concentration are then entirely due to changes in blood volume. Regular measurements of $\mathrm{X}$ concentration can therefore reveal the changes in blood volume. As it stands, however, this method provides estimates for intravascular volume and not interstitial volume or ICV. A more promising method, which does provide these two estimates, is multifrequency bioimpedance [13]. Here two electrodes are attached to the patient, typically one to the wrist and the other to the ankle, and the resulting electrode-electrode conductance is measured for a range of frequencies between $5 \mathrm{kHz}$ and $500 \mathrm{kHz}$, from which the total body water and extracellular volume can be calculated.

Our model is simpler than many other bicompartmental models in that it ignores the kinetics of urea or other solutes. This limits the model in providing a complete description of sodium profiling. For example, we cannot predict the onset of urea rebound following intercompartmental equilibration after the termination of dialysis [5] and we do not identify small transient water shifts which typically occur early in a dialysis session as the blood urea concentration rapidly falls and sets up a small IC:EC urea gradient. Apart from the limitation of the model in describing urea kinetics, the small number of dependent variables (solutes and fluid compartment volumes) in the model means that constraints are automatically placed upon its predictive scope. For example, as we have explained, the specification of the net quantity of sodium and water to be removed defines the final ECV and ICV. In models that include a second osmolyte, such as urea (e.g. [1] and [2]) the additional dependent variable (urea mass) introduces an extra degree of freedom so that the end of dialysis targets of ECV and EC sodium mass are no longer constrained as severely as in equation (25). One benefit of such models is that ECV and ICV may be more subtly controlled, in that the final ECV (and therefore also the ICV) is not simply determined by the required sodium and fluid adjustments. However, the development of a model consisting of a single differential equation for one unknown, namely extracellular sodium mass, from which the ICV and ECV can be calculated once the dialysate sodium and ultrafiltration profiles have been specified is an advantage. Also, the model can be reversed, and the dialysate sodium profile necessary to produce a desired extra- 
cellular sodium profile (and hence total sodium removed) for a given ultrafiltration profile can be determined.

\section{Acknowledgements}

We would like to acknowledge the assistance of the nursing and technical staff of the Mary Rankin Dialysis Unit of the Middlesex Hospital. SB is currently supported by a Wellcome Trust Mathematical Biology Research Training Fellowship.

\section{References}

[I] M. Ursino, L. Coli, M. Grilli Ciciloni, V. Dalmastri, A. Guidicissi, P. Masotti, G. Avanzolini, S. Stefoni, and V. Bonomini. A single mathematical model of intradialytic sodium kinetics: in vivo validation during hemodialysis with constant or variable sodium. Int. J. Artif. Organs 19 (6): 393-403, 1996.

[2] M. Coli, M. Ursino, V. Dalmastri, F. Volpe, G. La Manna, G. Avanzaloni, S. Stefoni and V. Bonomini. A simple mathematical model applied to selection of the sodium profile during proliled haemodialysis. Nephrol. Dial. Transplant. 13 $39-41,1998$.

13. M. G. Cogan and P. Schoenfield, (eds). Introduction to dialysis. Churchill Livingstone, New York, 1991.
[4] A.K. Cheung. Stages of future technological developments in haemodialysis. Nephrol. Dial. Transplant. 11 [Suppl. 8]: 5258. 1996.

[5] H. Mann and S. Stiller. Urea, sodium and water changes in profiling dialysis. Nephrol. Dial. Transplant. 11 [Suppl. 8]: 10-15, 1996.

[6] T.D. Kelly. Kinetics of intradialytic disequilibria: the problem. the causes, and new methods for the alleviation of patient morbidity. Nephrol. Dial. Transplant. 11 [Suppl. 8]: 3-9. 1996.

[7] J. E. Tattersall, P. Chamney, C. Aldridge, and R,N. Greenwood. Recirculation and the post-dialysis rebound. Nephrol. Dial. Transplant. 11 [Suppl. 2]: 20-23, 1996.

181 B. Redaelli. Electrolyte modelling in haemodialysis-potassium. Nephrol. Dial. Transplant. 11 [Suppl. 2]: 39-41, 1996.

[9] J. Robinson. A prelude to physiology. Blackwell Scientific Publications, 1981.

[10] O. Thews and H. Hutten. A comprehensive model of the dynamic exchanges during hemodialysis. Med. Progr. Technol. 16: $145-161,1990$

[11] J. A. Sargent and F. A. Gotch. Mathematical modelling of dialysis therapy. Kidncy Int. 18: [Suppl 10]: 2-10, 1980.

[12] V. Bonomini, L. Coli, G. Feliciangeli and M. P. Scolari. Biotechnology in profiled dialysis. Nephrol. Dial. Transplant. 11. [Suppl. 8]: 63-67, 1996.

$\lceil 13\rceil$ K. Katzarski, B. Charra, G, Laurant, F. Lopot, J.C. Divino-Filho, J. Nisell, and J. Bergstrom. Multifrequency bioimpedance in assessment of dry weight in haemodialysis. Nephrol. Dial. Transplant. 11 [Suppl. 2]: 20-23, 1996.

[14] A. Santoro, E. Mancini, F. Paolini, and P. Zucchelli. Blood volume monitoring and control. Nephrol. Dial. Transplant. 11 [Suppl, 2]: 20-23, 1996. 


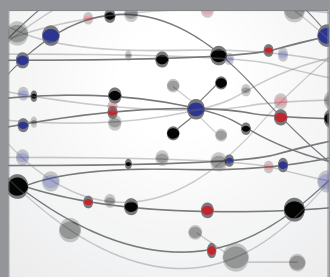

The Scientific World Journal
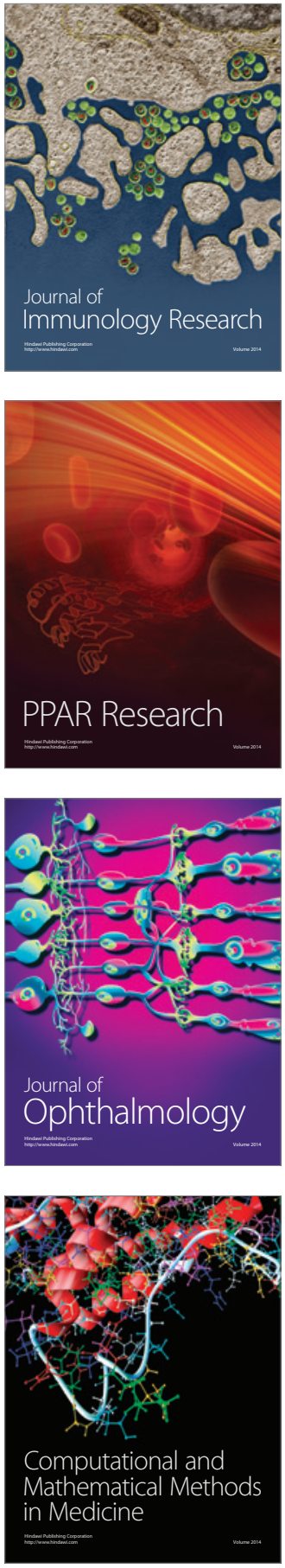

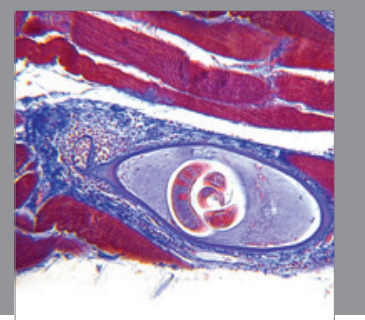

Gastroenterology

Research and Practice
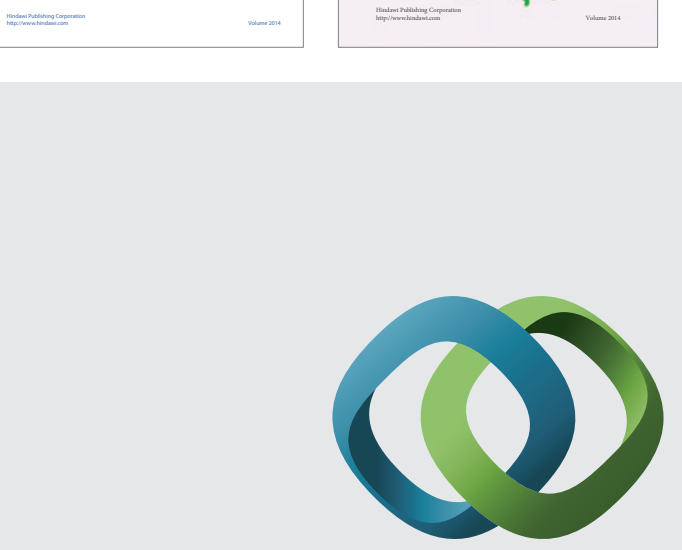

\section{Hindawi}

Submit your manuscripts at

http://www.hindawi.com
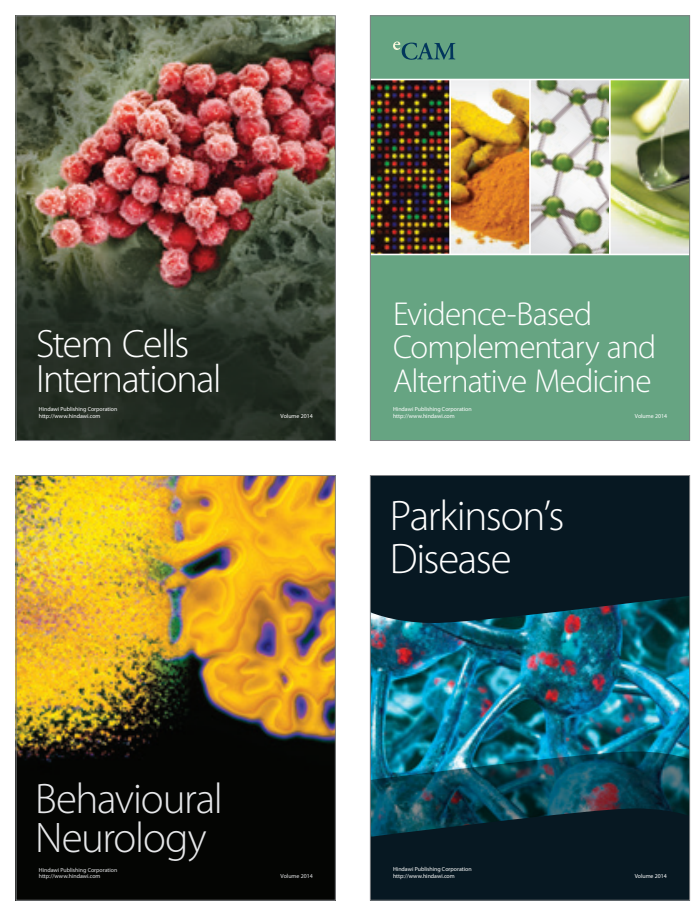

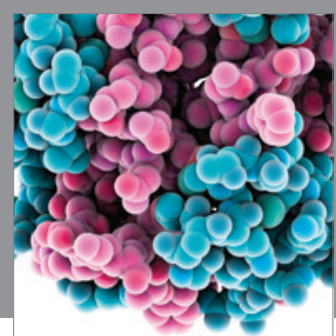

Journal of
Diabetes Research

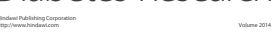

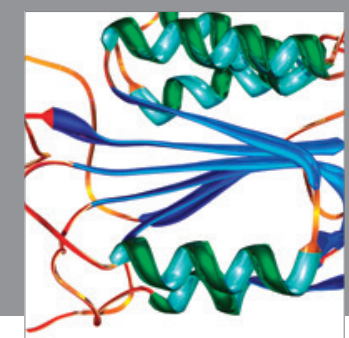

Disease Markers
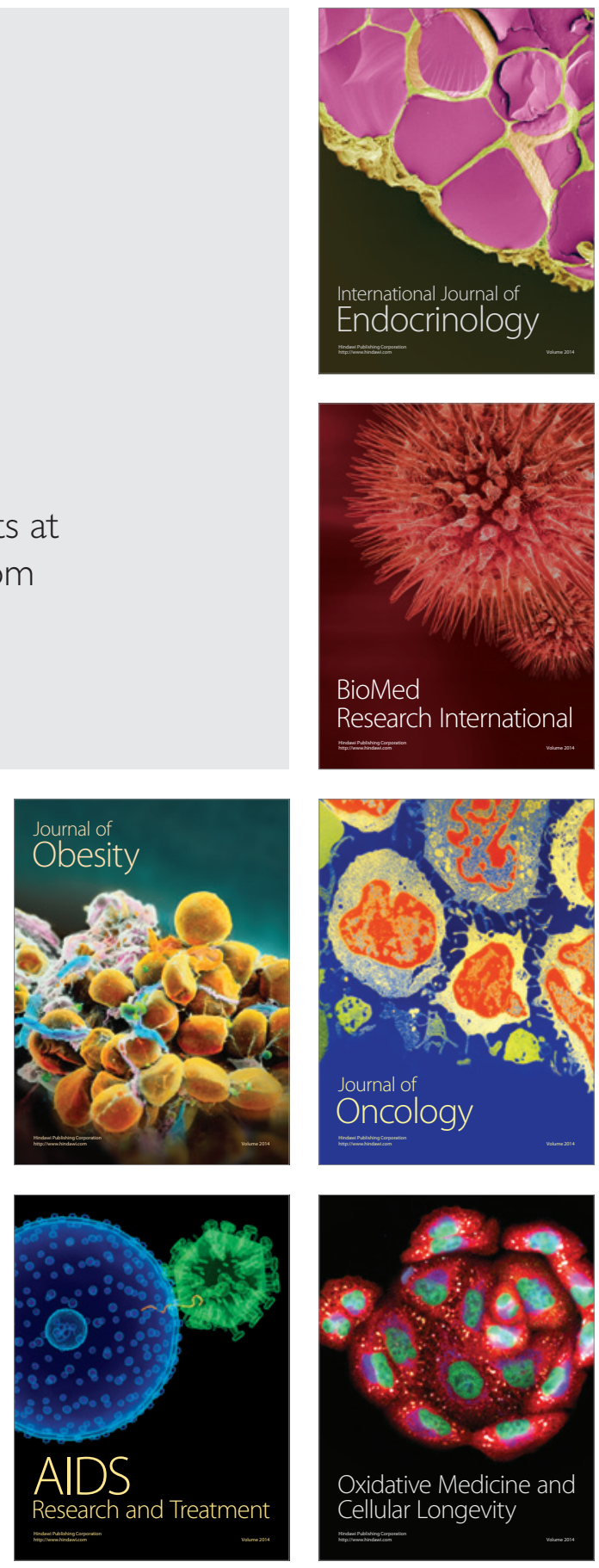